\title{
How to functionalise metal-organic frameworks to enable guest nanocluster embedment
}

Received 00th January 20xx, Accepted 00th January 20xx DOI: $10.1039 / \times 0 \times x 00000 x$

\author{
James King a , Linda Zhang ${ }^{b}$, Szymon Doszczeczko ${ }^{a}$, Olga Sambalovac, Hui Luo,d, Fadli Rohman ${ }^{\mathrm{e}}$, \\ Omotoyosi Phillips ${ }^{f}$, Andreas Borgschulte ${ }^{c}$, Michael Hirscher ${ }^{b}$, Matthew Addicoat ${ }^{g}$ and Petra Ágota \\ Szilágyi*a
}

\section{Introduction}

For heterogeneous catalysts, on which reactions only occur on surface atoms, a high surface-to-volume ratio is highly desirable ${ }^{1-3}$. This approach is even more sought after for catalysts consisting of precious metals such as Pd, whose reduction in size would be greatly beneficial economically. Nanoclusters are ultra-small particles that are typically on a scale of $\leq 1 \mathrm{~nm}$ for transition metals, consisting of fewer atoms than traditional nanoparticles, and boasting higher surface-tovolume ratios, as the vast majority of atoms are on the surface of transition metal nanoclusters ${ }^{4-6}$. The size of clusters on this scale have very different physical and chemical properties than bulk materials, or even nanoparticles ${ }^{7-10}$. Furthermore, the smaller the particles the more the 'contribution' of every atom matters, therefore it is crucial that clusters of uniform size can be obtained for any application ${ }^{11,12}$. The greatest challenge limiting our ability to design and synthesise large quantities of

School of Engineering and Materials Science, Queen Mary University of London, Mile End Campus, E1 4NS, London, United Kingdom.

b. Max Planck Institute for Intelligent Systems, Heisenbergstrasse 3, 70569 Stuttgart, Germany.

Empa Materials Science and Technology, Überland Strasse. 129, 8600 Dübendorf, Switzerland.

d. Department of Chemical Engineering, Imperial College London, South Kensington Campus, SW7 2AZ, London, United Kingdom.

e. Gemeinschaftslabor für Elektronenmikroskopie (GFE), RWTH Aachen University,

Ahornstr. 55, D-52074 Aachen, Germany.

f. University of Greenwich, Department of Pharmaceutical, Chemical and

Environmental Sciences, Chatham Maritime, ME4 4TB, United Kingdom.

gottingham Trent University - Clifton Campus, School of Science and Technology,

Clifton Lane Nottingham, Nottingham, NG11 8NS, United Kingdom.

* Author to whom correspondence should be sent p.szilagyi@qmul.ac.uk

Electronic Supplementary Information (ESI) available: [details of any supplementary

information available should be included here]. See DOI: 10.1039/x0xx00000x nanoclusters of identical composition and geometry is that the higher the surface-to-volume ratio the higher the surface energy, causing nanoclusters to be thermodynamically unstable ${ }^{13-15}$. This instability means that they are prone to stabilise themselves by sintering, a process in which larger clusters and particles are formed ${ }^{14}$. In order to adequately control the size and shape of nanoclusters, it is vital to prevent this from happening.

To stabilise nanoclusters one needs to employ a strongly binding support, typically 2D supports are applied, such as a facet of a metal-oxide crystallite or carbon surfaces, whose functionalities may strongly interact with a side of the nanoclusters ${ }^{4,16,17}$. However, as only the surface of such supports are useful the overall catalytically active surface area is typically reduced, which results in a smaller effective area. In addition, as only one side of the clusters is anchored, there are two phenomena that also need to be considered. Firstly, the metal-support interaction may control the metal particle geometry thereby controlling its activity and potentially selectivity in catalytic reactions. Secondly, this is a limited anchoring effect which might even be disrupted, particularly on recurrent chemical reactions that change the nanocluster electron density and consequently modulate the metal-support interaction, typically leading to the sintering of nanoclusters on catalyst cycling ${ }^{7,18}$. 3D porous supports on the other hand, that encapsulate the metal nanocluster anchoring it from multiple sides, do not have the same disadvantage; they have intrinsically higher surface areas and allow for the transport of liquids and gases from the host particle surface through its channels and pore apertures to the active sites ${ }^{19,20}$. Additionally, inclusion of the nanoclusters within the host pores 
may result in confinement phenomena shown to affect selectivity and enhance activity of heterogeneous catalysts via selective reactant adsorption, geometrically constraining the reaction and changes in surface energy of the catalyst ${ }^{21}$. It is highly important that the pores of such supports being on the scale of the desired nanocluster size, with a uniform size distribution throughout the material, i.e. the host should be crystalline and the pores should be part of the crystal structure. Moreover, to embed the clusters in the pores, strong host-guest interactions with the frameworks are required, similarly to $2 D$ supports. One such class of materials that would make promising 3D supports for nanoclusters are metal-organic frameworks (MOFs) ${ }^{14,22-25}$. These materials are built up of inorganic nodes interconnected by organic linkers which selfassemble into porous crystal lattices. The size and topology of the pores depend on the geometry and coordinative nature of the building units. An extensive number of these materials have emerged over the past couple of decades and their unique properties and variability make them viable for gas storage and separation $^{26,27}$, catalysis ${ }^{28,29}$, and in energy applications ${ }^{30-33}$, to name a few functions. The well-defined pore geometries of metal-organic frameworks is a consequence of their high crystallinity, which make them ideal supports for nanoobjects $^{34-36}$. The presence of the organic linkers and their high chemical modularity enable the functionalisation of the frameworks, which can allow for the necessary strong hostguest, 'anchoring', interactions with the guest particles to hold them in the pores ${ }^{36-41}$.

As well as the previously mentioned advantages that metalorganic frameworks have as 3D supports over their 2D counterparts, they also allow for precise size control of nanoclusters without the need for capping agents traditionally used in conjunction with 2D supports to enable some control over the particle size ${ }^{42}$; if they form within the pores of the material they cannot grow in size beyond these dimensions, as long as the conditions for the particle growth are milder and so the bonds holding together the MOF building blocks are maintained. Furthermore, embedding nanoclusters in MOFs could be of particular interest given the possibility to tailor the properties of the catalytically active clusters through controlling their geometry and surface chemistry, the latter of which has been demonstrated by some of $\mathrm{us}^{43}$. Strong host-guest interactions can occur between the clusters and the framework itself, with reports of these interactions between both the inorganic nodes ${ }^{44-46}$ and functional groups on the linkers ${ }^{46-50}$. These interactions can aid in both preventing aggregation of nanoclusters and increasing their selectivity for a desired product when used as catalysts ${ }^{51-56}$.

Although MOFs may seem to provide a facile way to control nanocluster sizes with their well-defined pore sizes, as well as provide beneficial interactions with the nanoclusters, guest embedment in pores remains a complex challenge as experience shows that larger particles can form on the surface of $\mathrm{MOFs}^{57-60}$. Given the dependence of the chemical and physical properties of nanoclusters on their size, for property control of transition-metal nanoclusters, it is imperative that the guest particles are embedded inside the MOF pores rather than decorating its surface. As such, a method to predict the success of embedding nanoclusters in a specific MOF would be a powerful tool when it comes to assessing potential supports. Some of us have previously put forward a simple method of predicting the success of embedding nanoclusters in the pores of MOFs, UiO-66 and $\mathrm{NH}_{2}$-UiO-66, by choosing the adsorption enthalpy of guest atoms on cut-outs of the MOF pores as model, which was confirmed by experiments ${ }^{43}$. In particular, the adsorption enthalpy of two single guest atoms was compared with a dimer of the guest, and the findings showed that both the overall adsorption enthalpy and the difference between the two scenarios can be or are responsible for the successful embedment. However, this simple approach and a small set of two Pd-MOF systems yielded two descriptors, i.e. adsorption enthalpy and difference in the adsorption enthalpies for individual guest atoms and dimers. Ideally, the model applied should yield a single descriptor for successful embedment, which would result in much more powerful predictions.

In the research we hereby report, we further refined our model for explaining and predicting the success of embedding nanoclusters in the pores of MOFs by enhancing our studies by looking at both tetrahedral and octahedral pores present in UiO-66 and by investigating a larger set of Pd-MOF systems. In addition, we explore differences in the electronic state of the nanoclusters in the frameworks with varying functionalities that suggest potentially beneficial host-guest interactions.

\section{Results and discussion}

Central to embedding nano-objects in the pores of metalorganic frameworks are the questions whether or not the embedding is successful, i.e. the guest particles be they nanoparticles, nanoclusters, or single atoms ${ }^{49}$ are inside the pores, and if they are, whether there is any strong interaction, such as charge transfer between the framework and the guest particles.

\section{Success of embedding.}

In our previous work ${ }^{43}$ the framework UiO-66 was selected as well as a functionalised analogue, $\mathrm{NH}_{2}-\mathrm{UiO}-66$, to demonstrate the model. Pd was chosen as the guest metal due to its catalytic relevance. A procedure was followed in which Density Functional Theory (DFT) calculations were later compared to experimental data. It was found that Pd nanoclusters were embedded in the amino-functionalised framework but not the pristine UiO-66. Higher adsorption enthalpy values and a lower energy difference between values for $\mathrm{Pd}$ as two isolated atoms or as a dimer suggest increased chances of embedding. $\mathrm{NH}_{2}-$ UiO-66 had higher values and only a difference of $4 \mathrm{~kJ} \mathrm{~mol}^{-1}$, i.e. within the error of the calculations, and the success of embedding Pd into its pores confirmed by experimental data. Advancing from this, the model was expanded to look in more 
detail by calculating adsorption enthalpy values in both the tetrahedral $\left(T_{h}\right)$ and octahedral $\left(\mathrm{O}_{h}\right)$ pores of the frameworks. We have chosen 3 additional functionalised analogues of the same framework to study; $(\mathrm{Cl})_{2}-\mathrm{UiO}-66, \mathrm{Br}-\mathrm{UiO}-66$ and $(\mathrm{OH})_{2^{-}}$ UiO-66.

To explore the Potential Energy Surface (PES) of Pd within each of the functionalised $\mathrm{Pd} \subset \mathrm{x}-\mathrm{UiO}-66$ analogues, a procedure broadly similar to our previous work was carried out. Firstly, coordinates of the tetrahedral and octahedral pores were cut from the crystal structure of pristine UiO-66. The phenyl linkers were then functionalised with selected functional groups, i.e. $\mathrm{Cl},-\mathrm{Br}$, and $-\mathrm{OH}$. In order to account for randomness in the orientation of functionalised linkers, due to their lower symmetry with respect to the unfunctionalised linker, several different functionalised models were produced. In each of these model pores, each of a single $\mathrm{Pd}$ atom ( $\mathrm{Pd})$, two single $\mathrm{Pd}$ atoms (separated by $>3 \AA$; $2 x P d$ ) and a Pd dimer $\left(\operatorname{Pd}_{2}\right)$, were stochastically placed using the Kick ${ }^{61}$ stochastic structure generator. A total of 166 structures were generated for the mono-functionalised linker (2-bromoterephthalate) and 176 structures were generated for the di-functionalised linkers (2,5dichloroterephthalate, 2,5-dihydroxylterephthalate). To ensure relevance of the cluster model to the periodic crystal, the coordinates of the ZrO cluster and the terminal carboxylate groups (which would connect to another ZrO cluster) were frozen in all optimisations. Binding energies were calculated as $\mathrm{E}(\mathrm{Pd} \subset \mathrm{x}-\mathrm{UiO}-66)-[\mathrm{E}(\mathrm{x}-\mathrm{UiO}-66)+n \mathrm{E}(\mathrm{Pd})]$, where $n$ is the number of $\mathrm{Pd}$ atoms placed in the pore. The highest resultant adsorption enthalpy values are summarised in Table 1 (and Table S2).

Br-UiO-66 had high values for adsorption, a relatively large decrease in adsorption enthalpy for dimeric $\mathrm{Pd}$ in the octahedral pore and a low difference in values for atomic and dimeric $P d$ in the $T_{h}$ pore, suggesting that embedment would be successful and sintering less energetically favourable. For $(\mathrm{Cl})_{2-}$ UiO-66, the adsorption enthalpy values for Pd as a dimer were significantly higher and the values themselves were low compared to the other analogues and it was predicted that embedding would not occur, as the formation of large $\mathrm{Pd}$ particles would be favoured in every scenario. On the other hand, in the case of $(\mathrm{OH})_{2}$-UiO-66, the adsorption enthalpy values are high for both pores (the highest figures calculated), however there is a large difference in between the atomic and dimeric values in favour of dimer formation. It was therefore stipulated that $(\mathrm{OH})_{2}$-UiO-66 offers a method to decrease the number of descriptors necessary to predict successful guest embedment to one. This $\mathrm{Pd}-(\mathrm{OH})_{2}-\mathrm{UiO}-66$ system should help determine whether it is the magnitude of the adsorption enthalpies or the difference in between the adsorption enthalpy of the individual atoms and the dimer that governs whether or not Pd would be embedded.
Table 1 Adsorption enthalpy of Pd atoms and dimers on MOFs

\begin{tabular}{|c|c|c|c|c|c|c|c|c|}
\hline \multirow[t]{2}{*}{$\begin{array}{l}\Delta H_{\text {ads }} \\
\mathrm{kJ} \mathrm{mol}^{-1}\end{array}$} & \multirow[t]{2}{*}{$\begin{array}{l}\text { UiO- } \\
66^{*}\end{array}$} & \multirow{2}{*}{$\begin{array}{l}\mathrm{NH}_{2}- \\
\mathrm{UiO}- \\
66^{*}\end{array}$} & \multicolumn{2}{|c|}{ Br-UiO-66 } & \multicolumn{2}{|c|}{$\begin{array}{l}(\mathrm{Cl})_{2} \text {-UiO- } \\
66\end{array}$} & \multicolumn{2}{|c|}{$\begin{array}{l}(\mathrm{OH})_{2^{-}} \\
\text {UiO-66 }\end{array}$} \\
\hline & & & $\mathrm{O}_{\mathrm{h}}$ & $T_{h}$ & $\mathrm{O}_{\mathrm{h}}$ & $T_{h}$ & $\mathrm{O}_{\mathrm{h}}$ & $T_{h}$ \\
\hline $\mathrm{Pd}, \mathrm{Pd}$ & 150 & 187 & 201 & 204 & 183 & 117 & 250 & 282 \\
\hline $\mathrm{Pd}_{2}$ & 173 & 191 & 160 & 214 & 193 & 157 & 346 & 352 \\
\hline
\end{tabular}

* Previously published values, please note that these values relate to the $T_{h}$ pores ${ }^{43}$

To verify the predictions made by the calculations, the three frameworks were synthesised by a solvothermal process following the work of Farha et al. $^{62}$ and Pd was embedded following the method detailed in our previous work ${ }^{43}$. The integrity of the framework after Pd-loading was verified using FT-IR spectroscopy (Figures S1 and S2), PXRD data collection (Figure S3), and BET isotherm measurements (Table S3). To compare the embedment of Pd nano-objects in the samples, High Angle Annular Dark Field-Scanning Transmission Electron Microscopy (HAADF-STEM) micrographs were obtained (Figure 1).

The micrograph of $\mathrm{Pd} \subset \mathrm{Br}$-UiO-66 shows no sign of what could be particles of $\mathrm{Pd}$ larger than the dimensions of the MOF pores with small dots of Pd corresponding to the pore dimensions, suggesting no Pd sintering and successful embedment in MOFs pores in agreement with the computational data. It is worth noting that such frameworks are challenging to image as they amorphise in the electron beam, while in the case of the $\mathrm{Pd} \subset \mathrm{Br}$ UiO-66 poor contrast further complicates the acquisition of high-resolution images. To assess the distribution of $\mathrm{Pd}$, we carried out high-resolution EDX mapping on the $\mathrm{Pd} \subset \mathrm{Br}-\mathrm{UiO}-66$ particles. The EDX analysis showed the presence of large amounts of well-dispersed Pd (Figure 2, and Figure S5), which, in combination with the lack of large Pd particles $(>1.5 \mathrm{~nm}$, on the MOF surface), should be discernible in the HAADF-STEM images. The presence of well-dispersed Pd nanoclusters, therefore demonstrates that the prediction for this system was indeed right and the embedding of $\mathrm{Pd}$ in the $\mathrm{Br}$-UiO-66 pores was successful.
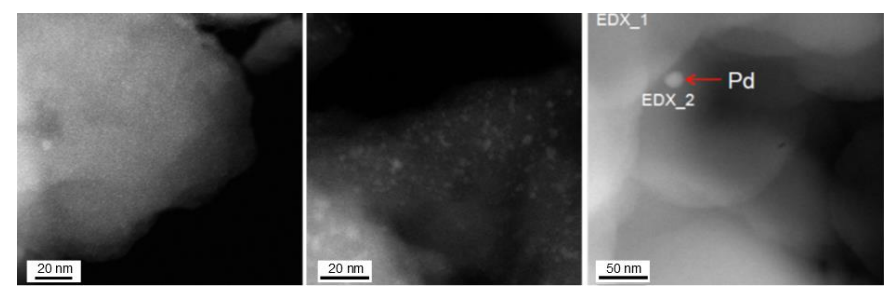

Figure 1 HAADF-STEM micrographs of PdCBr-UiO-66, $\mathrm{Pd} /(\mathrm{Cl})_{2}-\mathrm{UiO}-66$, and $\mathrm{Pd} /(\mathrm{OH})_{2-}$ UiO-66 (left to right). 

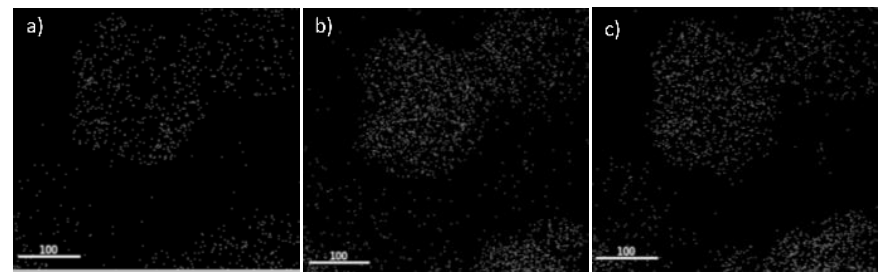

Figure 2 a) Reconstructed EDX maps of a) $\mathrm{Pd}, \mathrm{b}$ ) $\mathrm{Br}$ and c) Zr distribution within the MOF particles.

In the case of the $\mathrm{Pd} /(\mathrm{Cl})_{2}-\mathrm{UiO}-66, \mathrm{Pd}$ nanoparticles were observed in the HAAFD-STEM micrograph, as these particles of ca. 2-5 nm are larger than the pores of the MOF, they demonstrate that Pd nanoclusters were not embedded in the MOF pores, rather the sintering of $\mathrm{Pd}$ occurred and led to nanoparticle formation on the $(\mathrm{Cl})_{2}$-UiO-66 surface, which is in good agreement with the computational predictions. In the case of the $\mathrm{Pd} /(\mathrm{OH})_{2}-\mathrm{UiO}-66$ system, there was a distinct lack of $\mathrm{Pd}$ to be imaged. On a considerable effort, one large Pd particle was eventually discernible with no evidence of embedment of $\mathrm{Pd}$ in the pores of the MOF. This is an important finding as the modelling of Pd adsorption on $(\mathrm{OH})_{2}-\mathrm{UiO}-66$ promised a way of telling which of the two previously identified possible descriptors for the successful embedment of Pd in MOFs, i.e. magnitude of adsorption enthalpy or its difference for the adsorption of individual metal atoms or dimer, is valid. Relating the discovery that no Pd is embedded in the $(\mathrm{OH})_{2}$-UiO-66 pores back to the initial model, we conclude that it is the difference between adsorption enthalpies for the atomic and dimer $\mathrm{Pd}$ that is the most important descriptor when it comes to predicting the success of embedment.

\section{Pd adsorption sites and strong metal-support interactions.}

To further understand the reasons behind and ways in which Pd is embedded in the pores of the chosen MOFs, the preferred binding sites and motifs of each $\mathrm{Pd}_{n} \subset \mathrm{x}-\mathrm{UiO}-66$ can be examined. The lowest energy optimised structures of Pd, 2xPd and $\mathrm{Pd}_{2}$ in each $\mathrm{x}$-UiO-66 pore $\left(\mathrm{T}_{\mathrm{h}}\right.$ and $\left.\mathrm{O}_{\mathrm{h}}\right)$ were identified for further examination.

These structures (examples in Figure 3 ) reveal how Pd atoms interact with the functional groups on the frameworks and suggest how the host-guest interactions could occur.

In the case of $\mathrm{Pd \subset Br}-\mathrm{UiO}-66$, the preferential adsorption site of $\mathrm{Pd}$ is clearly on the $\mathrm{Br}$ atoms of the linker, implying strong host-
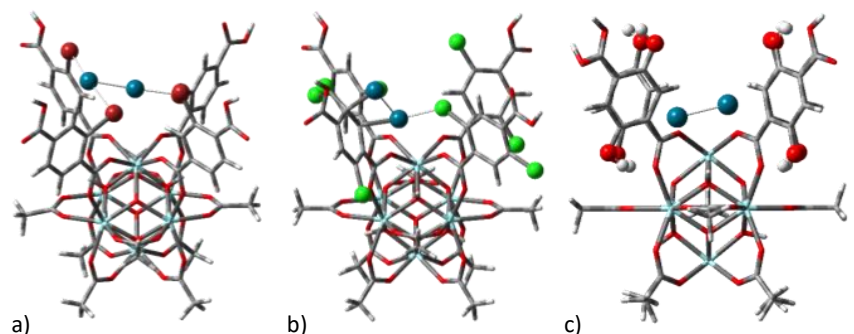

Figure 3 Models showing preferential adsorption sites for a $\mathrm{Pd}_{2}$ dimer on a) $\mathrm{Br}$-UiO-66, b) $(\mathrm{Cl})_{2}-\mathrm{UiO}-66$, and c) $(\mathrm{OH})_{2}-\mathrm{UiO}-66$. The representation of the depicted elements is as follows; $\mathrm{Pd}$ - blue ball; $\mathrm{Zr}$ - turquoise stick; $\mathrm{C}$ - grey stick; $\mathrm{O}$ - red stick and red ball (in c)); $\mathrm{H}$ - white stick; $\mathrm{Br}$ - burgundy ball; and $\mathrm{Cl}$ - green ball. guest interactions. On the other hand, $\mathrm{Pd} /(\mathrm{Cl})_{2}-\mathrm{UiO}-66$, the majority of interactions take place on the weaker adsorption sites of the benzene rings, which is consistent with weak hostguest interactions, and consequent sintering of Pd. Finally, in the $\mathrm{Pd} /(\mathrm{OH})_{2}-\mathrm{UiO}-66$ model, no interactions between the framework and the $\mathrm{Pd}$ are discernible, which is in line with the above observation of unsuccessful Pd embedding, probably on account of a lack of strong anchoring by the host.

As we previously demonstrated that the $\mathrm{Pd}$ nanoclusters are embedded in the pores of $\mathrm{Br}-\mathrm{UiO}-66$, and as our modelling predicts strong host-guest interactions, such interactions should alter the chemical state of both guest and host significantly enough to be measurable, similarly to what has been observed in the case of $\mathrm{NH}_{2}$-UiO-66. X-ray Photoelectron Spectroscopy (XPS) was used to detect changes in the chemical state of guest $(\mathrm{Pd})$ and host, i.e. the elements of the functional groups: $\mathrm{Br}, \mathrm{Cl}$, and $\mathrm{O}$ (Figure 4 ).

Figure 4 clearly demonstrates that the presence and chemical state of Pd significantly differs between the three samples. It should be noted, that this region overlaps with the $3 p$ region of $\mathrm{Zr}$, and the two intense peaks at $c a .333 .5$ and $347.1 \mathrm{eV}$ correspond to $\mathrm{Zr} 3 p_{3 / 2}$ and $\mathrm{Zr} \quad 3 p_{1 / 2}$ binding energies, respectively. The $\mathrm{Pd}$ signal (only the $3 d_{3 / 2}$ can be clearly resolved as the $3 d_{5 / 2}$ is convoluted with the $\mathrm{Zr} 3 p_{3 / 2}$ peak) is centred at $341.0 \mathrm{eV}$. The most obvious observation is the clear lack of a Pd signal in the case of the $(\mathrm{OH})_{2}-\mathrm{UiO}-66 \mathrm{MOF}$, this was underpinned by our difficulties to image the Pd particles in this framework and is in line with the lack of model interactions between framework and the Pd atoms (Figure 2c). It is also apparent that two different $\mathrm{Pd}$ species are observed in the $\mathrm{Br}$ UiO-66: referring to $\mathrm{Pd}$ and an oxidised $\mathrm{Pd}$ species, centred at $341.1 \mathrm{eV}$ and $342.5 \mathrm{eV}$, respectively. No oxidised Pd is observed in case of $(\mathrm{Cl})_{2}$-UiO-66. In fact, our data demonstrates that the $\mathrm{Pd}$ binding energy on the $(\mathrm{Cl})_{2}$-UiO-66 host corresponds well to that of the bulk Pd metal, similarly to what was observed for the pristine UiO-66 ${ }^{43}$, and in line with weak interactions. On the other hand, the $\mathrm{Pd}$ binding energy of the Pd nanoclusters embedded in the Br-UiO-66 framework is higher, revealing a degree of surface oxidation. The changes in binding energy of
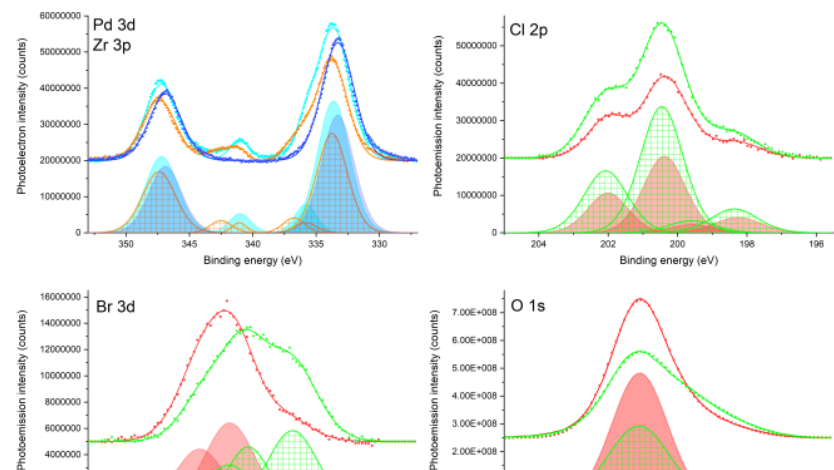

Figure 4 High resolution XPS spectra of $\mathrm{Pd} /(\mathrm{Cl})_{2}-\mathrm{UiO}-66$ (cyan), $\mathrm{Pd} \subset \mathrm{Br}$-UiO-66 (orange) and $\mathrm{Pd} /(\mathrm{OH})_{2}-\mathrm{UiO}-66$ (blue). The $\mathrm{Cl} 2 p, \mathrm{Br} 3 d$ and $\mathrm{O} 1 s$ region depict the empty MOFs (red) and the Pd-laden samples (green). For the details of the fitting refer to Table S4 of supplementary information. 
Table 2 Summary of the XPS data, showing $\Delta E_{\mathrm{b}}$ values for the functional groups with respect to the empty framework and embedded Pd compared with metallic Pd.

\begin{tabular}{|c|c|c|c|}
\hline MOF & Species & $\begin{array}{c}\Delta E_{b} \mathrm{w} \& \mathrm{w} / \mathrm{o} \\
\quad \mathrm{Pd}(\mathrm{eV})\end{array}$ & $\begin{array}{c}\Delta E_{b} \text { Pd wrt. bulk } \\
\text { (eV) }\end{array}$ \\
\hline UiO-66* & $n / a$ & $n / a$ & 0.0 \\
\hline $\mathrm{NH}_{2}$-UiO-66* & $\mathrm{N} 1 \mathrm{~s}$ & -0.3 & +0.7 \\
\hline$(\mathrm{Cl})_{2}-\mathrm{UiO}-66$ & $\mathrm{Cl} 2 p$ & 0.0 & 0.0 \\
\hline Br-UiO-66 & $\mathrm{Br} 3 \mathrm{~d}$ & -1.0 & +1.1 \\
\hline$(\mathrm{OH})_{2}-\mathrm{UiO}-66$ & 0 1s & $n / a$ & $n / a$ \\
\hline
\end{tabular}

*Previously published values ${ }^{43}$

both the $\mathrm{Pd}$ and the elements in the functional groups are summarised below alongside previously published values for

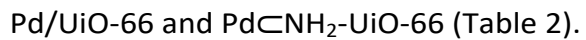

As such small nanoclusters are highly thermodynamically unstable on account of their large surface energy, it is imperative to establish whether the surface oxidation is a consequence of strong-host-guest interactions, as suggested by the computational modelling, or through a small degree of reaction with moisture or air, by evaluating the binding energy of the functional group with which the adsorbed $\mathrm{Pd}$ can interact. In the case of Br-UiO-66, and according to our computational model, this element should be $\mathrm{Br}$. And indeed we detect a shift in the $\mathrm{Br} 3 d$ binding energy towards lower values when compared with the empty $\mathrm{Br}$-UiO-66, going from predominantly "organic" $\mathrm{Br}\left(\mathrm{Br} 3 d_{5 / 2}\right.$ centred at $\left.70.7 \mathrm{eV}\right)$ to mostly "inorganic" $\mathrm{Br}$ state $\left(\mathrm{Br} 3 d_{5 / 2}\right.$ centred at $\left.68.8 \mathrm{eV}\right)$, which is line with a Br-Pd interaction. Such binding energy shifts for $\mathrm{Cl}$ $2 p$ in $(\mathrm{Cl})_{2}-\mathrm{UiO}-66$ vis-à-vis $\mathrm{Pd} /(\mathrm{Cl})_{2}-\mathrm{UiO}-66$ and for $\mathrm{O} 1 \mathrm{~s}$ in $(\mathrm{OH})_{2}-$ UiO-66 vis-à-vis $\mathrm{Pd} /(\mathrm{OH})_{2}$-UiO-66 have not been observed. It can be therefore concluded that the oxidation of Pd observed in the $\mathrm{Pd} \subset \mathrm{Br}$-UiO-66 sample is a consequence of its strong interaction with the $-\mathrm{Br}$ functional group.

It should be emphasised that in all cases, our computational predictions have been in excellent agreement with our experimental observations with XPS.

We note that when comparing the degree of surface oxidation via host-guest interactions in $\mathrm{Pd} \subset \mathrm{NH}_{2}-\mathrm{UiO}-66$ and $\mathrm{Pd} \subset \mathrm{Br}-\mathrm{UiO}$ 66 , the $\mathrm{Pd}$ embedded in $\mathrm{Br}$-UiO-66 appears to be more oxidised (Table 2). This could result in a different level of catalytic activity. In order to test this, we have selected $\mathrm{H}_{2}-\mathrm{D}_{2}$ isotope scrambling as a simple reaction to verify if there is a catalytic activity difference related to the slight surface chemistry difference in these Pd nanoclusters embedded in functionalised UiO-66 MOFs. The reaction progress can be monitored on the simple recognition that as the thermodynamic stability of $\mathrm{H}_{2}$ and $D_{2}$ is equal, and as the reaction takes place through the steps of physisorption of the diatomic molecules, followed by the splitting of the H-H or D-D bonds, recombination of the atoms into diatomic molecules, and eventual desorption, the molecule of composition HD should also form. Furthermore, if the reaction mixture contained $\mathrm{H}_{2}: \mathrm{D}_{2}$ in a $1: 1$ ratio, the composition of the gas phase should reach $\mathrm{H}_{2}: H D: D_{2}$ of $1: 2: 1$ in

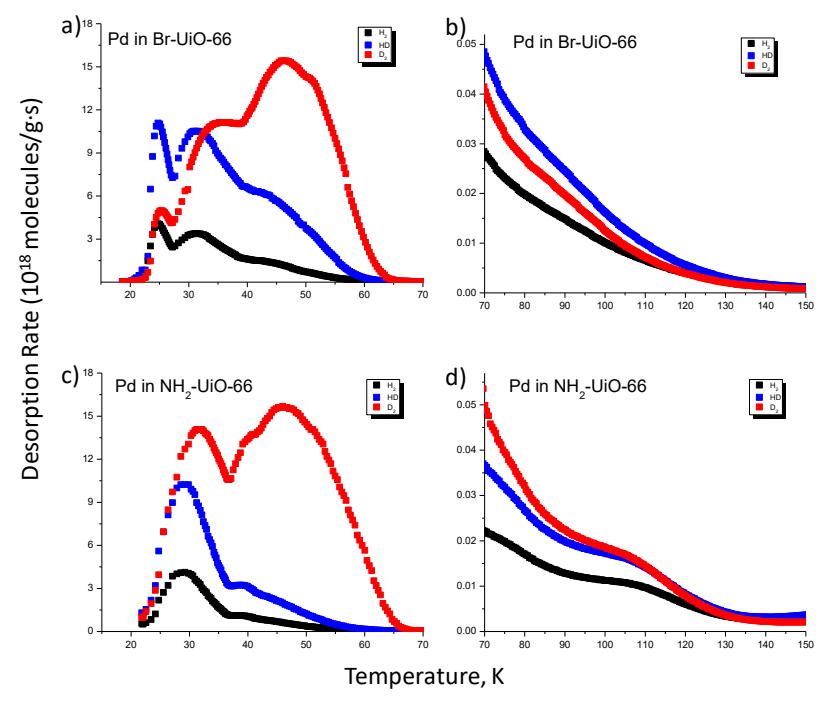

Figure 5 Thermal desorption spectra for $\mathrm{H}_{2}-\mathrm{D}_{2}$ isotope scrambling reaction mixture allowed on Pd-containing MOFs at ambient temperature for 30 minutes, spectra indicate $\mathrm{Pd} \subset \mathrm{Br}-\mathrm{UiO}-66 \mathrm{a}$ ) and b) and $\mathrm{Pd} \subset \mathrm{NH}_{2}-\mathrm{UiO}-66$; c) and d) catalysts at two distinct desorption temperature regions.

dynamic equilibrium. Thermal desorption spectroscopy (TDS) can be used to analyse the gas phase composition ${ }^{63}$ bearing in mind that the adsorption enthalpy of the different isotopes differs on the same framework, and their adsorption kinetics also differs on account of the different kinetic energies, which is a consequence of their different molecular masses. Taking these limitations into account, it is allowed to assume that any difference in the TDS spectra of $\mathrm{Pd} \subset \mathrm{NH}_{2}-\mathrm{UiO}-66$ and $\mathrm{Pd} \subset \mathrm{Br}$ UiO-66 can be assigned to differences in the $\mathrm{Pd}$ surface chemistry, provided that spectra were acquired under the same conditions and that the $\mathrm{Pd}$ particle sizes are identical. We therefore carried out a careful analysis of the $\mathrm{H}_{2}-\mathrm{D}_{2}$ isotope scrambling reaction on $\mathrm{Pd} \subset \mathrm{NH}_{2}-\mathrm{UiO}-66$ and $\mathrm{Pd} \subset \mathrm{Br}-\mathrm{UiO}-66$ (Figure 5), when the reactants were mixed at $20 \mathrm{~K}$ and ambient temperature, and in comparison with the empty MOFs (Figures S6 and S7, ESI).

In order to better observe the catalytic activity, the desorption spectra were analysed in two distinct temperature regions; in the low-temperature region up to $70 \mathrm{~K}$, physisorbed gas molecules are typically desorbing from the pore surfaces. In case of an isotopic gas mixture, heavier molecules are preferentially adsorbed owing to the mass dependence of the zero-point energy, i.e. $\mathrm{D}_{2}$ highest signal and if scrambling has occurred $\mathrm{D}_{2}>\mathrm{HD}>\mathrm{H}_{2}$ signal intensity is expected. In the following region between $70-150 \mathrm{~K}$, stronger interactions with the frameworks may be probed ${ }^{64}$.

For spectra acquired after exposing the MOF with a $\mathrm{H}_{2} / \mathrm{D}_{2}$ isotope mixture at $20 \mathrm{~K}$, the evolution of all gas molecules follows exactly the same trend, which is consistent with no isotope scrambling reaction taking place at such low temperatures (Figure S6, ESI). On the other hand, the spectra acquired when the isotope mixture reacts at ambient temperatures with the MOF, clearly shows that HD molecules 
have been formed only in the Pd-doped UiO-66 (Figure 5), demonstrating that both catalysts are active under the applied conditions. It is important to note that HD formation was not observed in the 'empty' Br-UiO-66 and $\mathrm{NH}_{2}-\mathrm{UiO}-66$ MOFs (Figure S7, ESI).

However, it is apparent that the desorption spectra differ in both temperature ranges, furthermore, their deviation from the desorption spectra of the 'empty' MOFs is also different. Particular differences include the additional desorption peak off PdCBr-UiO-66 centred at ca. $25 \mathrm{~K}$, which most likely can be attributed to desorption from the pores, and the additional shoulder in $\mathrm{Pd} \subset \mathrm{NH}_{2}-\mathrm{UiO}-66$, centred around $105 \mathrm{~K}$. We were unable to explain these observations with a slight activity change expected to arise on account of a different surface chemistry of the Pd nanocluster catalysts, which would have been expected to only modulate the concentration of the gas mixture. In order to investigate this further, we considered the original hypotheses on the validity of any comparison between the desorption spectra of the two systems, i.e. it provides a valid comparison as long as the experimental conditions are the same and the particle sizes are the same. The experiments were designed so that the first condition would be met. However, it was possible that we have overlooked some discrepancies in the $\mathrm{Pd}$ nanocluster size, particularly for the $\mathrm{Pd} \subset \mathrm{Br}-\mathrm{UiO}-66$, where the low contrast and tendency of the host framework to amorphise under beam rendered imaging very challenging.

It was therefore for the above reason that we have further collected a large number of HAADF-STEM micrographs on the $\mathrm{Pd} \subset \mathrm{Br}-\mathrm{UiO}-66$ system, and indeed found that there appear to be some amorphous $\mathrm{Br}-\mathrm{UiO}-66$ regions in the specimen (Figure 6). As these regions are not crystalline they don't contain the same porous structures and as such they are not able to act as an effective template for nanocluster growth within their pores. As a consequence, $\mathrm{Pd}$ nanoparticles of $c a .5 \mathrm{~nm}$ formed on the surface of the amorphous region, which also means that the TDS spectra are not directly comparable for the two catalyst systems. This also explains the observation of metallic Pd in the XPS spectrum. It should be noted that the appearance of the

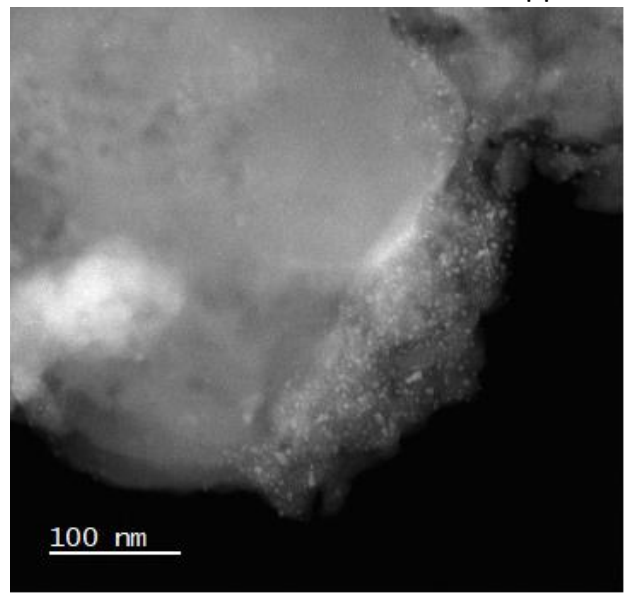

Figure 6 HAADF-STEM micrograph of PdCBr-UiO-66 showing an amorphous region with a high number of larger Pd particles. additional low-temperature desorption peak in the TDS spectrum of $\mathrm{Pd} \subset \mathrm{Br}-\mathrm{UiO}-66$ can be explained by the increased porosity of the $\mathrm{Br}-\mathrm{UiO}-66$ on addition of $\mathrm{Pd}$, where the additional pores are those between the MOF and Pd particles.

While our experiments reveal that the concentration of these amorphous regions is low, their existence is sufficient to skew the catalysis data and therefore we were unable to unequivocally demonstrate whether the different surface chemistry of $\mathrm{Pd}$ nanoclusters embedded in functionalised metal-organic frameworks can have a significant impact on their catalytic properties.

\section{Conclusions}

We have developed and tested an enhanced computational model capable of robust predictions related to the successful embedding of guest particles into the pores of metal-organic frameworks. This simple model takes into consideration all types of MOF pores and has the potential to be employed for a variety of guest materials. The above computational model was tested on a set of 3 functionalised frameworks, and was found to provide robust predictions. Importantly, we have been able to reduce the number of descriptors for successful guest particle embedding into MOFs to one, i.e. the difference in adsorption enthalpies of individual guest atoms with respect to that of their clustered counterparts.

Using the above predictions, we were able to embed $\mathrm{Pd}$ nanoclusters in the pores of $\mathrm{Br}-\mathrm{UiO}-66$, and demonstrate the strong host-guest interactions that anchor the guest nanoclusters inside the frameworks. Interestingly, only monofunctionalised linkers allowed for the embedding of $\mathrm{Pd}$ nanoclusters in the MOF pores (2-bromoterephthalate and 2aminoterephthalate) as opposed to the unfunctionalised (terephthalate) and bifunctionalised (2,5-dichloroterephthalate and 2,5-dihydroxylterephthalate) ones. Pd nanoclusters anchored in the pores of MOFs with different functionalities, i.e. $-\mathrm{NH}_{2}$ and $-\mathrm{Br}$, have a different degree of interaction strength with the frameworks, directly translating into a different level of surface modification of the guest particles. This approach may be directly exploited to tune the catalytic activity of supported nanoclusters. Finally, we have developed a robust approach for the design of such systems and a tool box for their characterisation, which will promote the exploitation of surface modification of nanoclusters via their embedding into functionalised metal-organic framework pores.

\section{Experimental}

All reagents and solvents are commercially available and were used without further purification.

\section{Synthetic procedures.}

All MOF syntheses were carried out following the method developed by Farha et al. ${ }^{62}$. The procedure described used 
amounts of reagents given in Table S1. A $25 \mathrm{ml}$ reaction vial was loaded with $\mathrm{ZrCl}_{4}$ and one third of the DMF, to which the concentrated $\mathrm{HCl}$ was added before sonication to fully dissolve the $\mathrm{ZrCl}_{4}$. The ligand was dissolved in the remainder of the DMF, which was then added to the $\mathrm{ZrCl}_{4}$ mixture before being heated at $80^{\circ} \mathrm{C}$ overnight. The product precipitated out of solution and was filtered by a centrifugation procedure, washed with DMF twice and then washed with $\mathrm{EtOH}$ twice. The samples were activated in-vacuo at $125^{\circ} \mathrm{C}$ overnight.

For Pd loading, $10 \mathrm{mg}, 0.049 \mathrm{mmol}$ of the precursor $\left(\mathrm{Pd}\left(\mathrm{NO}_{3}\right)_{2} \cdot 2 \mathrm{H}_{2} \mathrm{O}\right)$ was dissolved in $7 \mathrm{ml}$ of anhydrous acetonitrile before being added to the prepared $100 \mathrm{mg}$ MOF under an inert atmosphere. This produced samples with a nominal $10 \mathrm{wt} \%$ guest loading, which was gently heated and stirred $\left(50^{\circ} \mathrm{C}\right.$ for 24 hours) before being filtered and washed with acetonitrile. The reduction of the Pd precursor was carried out in a $5 \%$ hydrogen in argon stream ( 3 hours, $150{ }^{\circ} \mathrm{C}$ ) using a custom-made cell in a tube furnace.

\section{Calculations.}

All calculations were carried out using the AMS software ${ }^{65}$ with the PBE-D366-68 exchange-correlation potential and scalar relativity with the TZ2P basis set and a small frozen core. Constrained geometry optimisations were undertaken with atoms corresponding to the ZrO cluster and the carboxylate groups of the BDC linkers frozen at the crystallographic positions, all other atoms were optimised.

\section{Thermal desorption spectroscopy (TDS).}

TDS experiments were carried out on an in-house designed device with about $2 \mathrm{mg}$ of each sample. The sample holder is screwed tightly to a Cu block, which is surrounded by a heating spiral in the high vacuum chamber. The Cu block is connected to a flowing helium cryostat, allowing cooling below $20 \mathrm{~K}$. All the samples were first loaded in the sample holder and activated at $420 \mathrm{~K}$ under vacuum for $2 \mathrm{~h}$. Then, the sample was exposed to a 50 mbar equimolar $D_{2} / H_{2}$ isotope mixture at room temperature for $30 \mathrm{~min}$. Afterwards, the sample was rapidly cooled down below $20 \mathrm{~K}$, and the gas molecules that had not been adsorbed were pumped out. Then a linear heating ramp $(0.1 \mathrm{~K} / \mathrm{s})$ was applied. In the other case, the activated sample was exposed to a $50 \mathrm{mbar} \mathrm{D}_{2} / \mathrm{H}_{2} 1: 1 \mathrm{mixture}$ at $20 \mathrm{~K}$ for $30 \mathrm{~min}$. Different from exposure at room temperature, the remaining gas molecules were removed mildly at $20 \mathrm{~K}$ until high vacuum was reached again. Afterwards, the sample was cooled down to the boiling temperature. Finally, during heating from $20 \mathrm{~K}$ to room temperature with a heating rate of $0.1 \mathrm{~K} / \mathrm{s}$, the desorbing gas was continuously detected using a mass spectrometer (QMS), recognizing a pressure increase in the sample chamber when gas desorbs. The area under the desorption peak was proportional to the desorbing amount of gas, which can be quantified after careful calibration of the TDS apparatus.
Calibration of the mass spectrometer signal: A solid piece of a diluted Pd alloy $\mathrm{Pd}_{95} \mathrm{Ce}_{5}(\sim 0.5 \mathrm{~g})$ was used for calibration. Before the calibration, the oxide layer of the alloy was removed by etching with aqua regia. Then the alloy was heated up to $600 \mathrm{~K}$ under high vacuum to remove any hydrogen that might be absorbed during the etching procedure. Afterwards, it was exposed to 40 mbar pure $\mathrm{H}_{2}$ or pure $\mathrm{D}_{2}$ for $1.5-2.5 \mathrm{~h}$ at $350 \mathrm{~K}$ after the mass had been collected. As $\mathrm{H}$ and $\mathrm{D}$ were bound preferentially to the Cerium atoms at low exposure pressures, the alloy could be handled under ambient conditions for a short time. The alloy was weighed after being cooled down to room temperature. The mass difference between unloaded state and loaded state was equal to the mass uptake of hydrogen or deuterium, respectively. After weighing, the alloy was loaded in the chamber again, and then a $0.1 \mathrm{~K} / \mathrm{s}$ heating ramp (RT to 600 K) was applied for a subsequent desorption spectrum. The obtained mass of gas is directly corresponded to the area under the desorption peak.

\section{X-ray Photoelectron Spectroscopy (XPS).}

XPS was measured under ultrahigh vacuum $\left(<5 \times 10^{-7} \mathrm{~Pa}\right)$ on a Quantum 2000 (Physical Electronics Inc.) instrument, equipped with an Al monochromatic X-ray source (photon energy = $1486.7 \mathrm{eV}$ ). The data was analysed by the CasaXPS software. Peaks were fitted with a numerical convolution of a Lorentzian with a Gaussian lineshapes GL (30), after the Shirley background subtraction. The peak positions are summarised in Table S4 of supplementary information.

\section{Fourier Transform Infrared (FTIR) Spectroscopy.}

FTIR spectroscopy was carried out using an attenuated total reflectance (ATR) setup using a Bruker Tensor 27 spectrometer. A resolution of $4 \mathrm{~cm}^{-1}$ was used with a range of $400-4000 \mathrm{~cm}^{-1}$, with 32 scans. The software used to record the spectra was OPUS.

\section{Powder X-ray Diffraction (PXRD).}

PXRD data was collected on a Panalytical X'Pert Pro in reflection mode using a $\mathrm{Cu} K_{\alpha}$ anode $(\lambda=1.54178 \AA$ A), divergence slit, $\mathrm{Ni}$ filter and a range of $5-120^{\circ} 2 \Theta$.

\section{Low-pressure gas-sorption measurements.}

Low pressure nitrogen physisorption isotherms were measured at $77 \mathrm{~K}$ using a liquid nitrogen bath on a Quantachrome NOVA4200e Surface Area and Pore Size Analyzer. The software used was QuantaChrome NovaWin. Isotherm points were chosen to be able to determine the BET (Brunauer-EmmettTeller) surface area and pore volume.

\section{Scanning Transmission Electron Microscopy (STEM).}

HAADF-STEM micrographs were obtained for PdCBr-UiO-66 using a JEOL ARM300CF operating at $300 \mathrm{kV}$ at ePSIC, at Diamond Light Source. 
Micrographs for $\mathrm{Pd}$ in $(\mathrm{OH})_{2}$-UiO-66 were obtained using a FEI TEM Tecnai F2O accelerated at $200 \mathrm{kV}$ with field-emission gun (FEG) and equipped with Gatan GIF 2000 energy filter, XEDS (EDAX) and STEM-HAADF detectors (Fichione).

\section{Energy Dispersive X-ray (EDX) Spectroscopy.}

EDX was performed on the $\mathrm{Pd} \subset \mathrm{Br}$-UiO-66 sample using an Oxford Instruments XMAX 100 EDX detector in tandem with HAADF-STEM micrographs obtained at ePSIC, Diamond Light Source. The files were processed to produce the spectra using HyperSpy.

EDX was performed on $\mathrm{Pd}$ in $(\mathrm{OH})_{2}-\mathrm{UiO}-66$ using a FEI TEM Tecnai F20 accelerated at $200 \mathrm{kV}$ with Gatan GIF 2000 energy filter, XEDS (EDAX) and STEM-HAADF detectors (Fichione).

Inductively Coupled Plasma Mass Spectrometry (ICP-MS). $\mathrm{Pd}$ content of samples was analysed by ICP-MS using $\mathrm{A} \mathrm{Nu}$ Instruments Nu Plasma multiple collector inductively coupled plasma mass spectrometer. Before analysing, the MOF matrix was carefully weighed and digested aqua regia using a MARS 6 microwave at $1500 \mathrm{~W}$ for $5 \mathrm{~min}$. The solutions were diluted with $2 \% \mathrm{HNO}_{3}$ and $1 \% \mathrm{HCl}$ to achieve a concentration of $\mathrm{Zr}^{4+}<1 \mathrm{ppm}$. For each sample, two duplicates were prepared for accuracy. External Pd standard solutions were prepared by diluting a commercially available standard of $1003 \mathrm{mg} / \mathrm{g}$ to 5, 10, 50, 100 ppb with $2 \% \mathrm{HNO}_{3}$ and $1 \% \mathrm{HCl}$.

\section{Conflicts of interest}

There are no conflicts to declare.

\section{Acknowledgements}

PAS would like to thank the support of the EPSRC (grant \# EP/N00938X/1). MAA thanks the Materials Chemistry Consortium (EPSRC EP/L000202, EP/R029431) for HPC resources, this work used the UK Materials and Molecular Modelling Hub, which is partially funded by EPSRC (EP/P020194). This work was partly supported by the UZH-UFSP program LightChEC. Financial support from the Swiss National Science Foundation (grant number 172662) is greatly acknowledged.

\section{Notes and references}

1 J. Lin, B. Qiao, J. Liu, Y. Huang, A. Wang, L. Li, W. Zhang, L. F. Allard, X. Wang and T. Zhang, Angew. Chemie - Int. Ed., 2012, 51, 2920-2924.

2 A. A. Herzing, C. J. Kiely, A. F. Carley, P. Landon and G. J. Hutchings, Science (80-. )., 2008, 321, 1331-1335.

3 K. L. Kelly, E. Coronado, L. L. Zhao and G. C. Schatz, J. Phys. Chem. B, 2003, 107, 668-677.

4 M. Imran, A. B. Yousaf, X. Zhou, Y. F. Jiang, C. Z. Yuan, A. Zeb, N.
Jiang and A. W. Xu, J. Phys. Chem. C, 2017, 121, 1162-1170.

5 J. P. Wilcoxon and B. L. Abrams, Chem. Soc. Rev., 2006, 35, 1162-1194.

6 C. Pan, K. Pelzer, K. Philippot, B. Chaudret, F. Dassenoy, P. Lecante and M. J. Casanove, J. Am. Chem. Soc., 2001, 123, 7584-7593.

7 T. W. Hansen, A. T. Delariva, S. R. Challa and A. K. Datye, Acc. Chem. Res., 2013, 46, 1720-1730.

8 X. Zhang, H. Shi and B. Q. Xu, Angew. Chemie - Int. Ed., 2005, 44, 7132-7135.

9 W. Tang, Z. Hu, M. Wang, G. D. Stucky, H. Metiu and E. W. McFarland, J. Catal., 2010, 273, 125-137.

10 S. Sun, G. Zhang, N. Gauquelin, N. Chen, J. Zhou, S. Yang, W. Chen, X. Meng, D. Geng, M. N. Banis, R. Li, S. Ye, S. Knights, G. A. Botton, T. K. Sham and X. Sun, Sci. Rep., , DOI:10.1038/srep01775.

11 J. M. Thomas, Z. Saghi and P. L. Gai, Top. Catal., 2011, 54, 588594.

12 B. Qiao, A. Wang, X. Yang, L. F. Allard, Z. Jiang, Y. Cui, J. Liu, J. Li and T. Zhang, Nat. Chem., 2011, 3, 634-641.

13 H. R. Moon, D. W. Lim and M. P. Suh, Chem. Soc. Rev., 2013, 42, 1807-1824.

14 C. T. Campbell, Acc. Chem. Res., 2013, 46, 1712-1719.

15 X. F. Yang, A. Wang, B. Qiao, J. Li, J. Liu and T. Zhang, Acc. Chem. Res., 2013, 46, 1740-1748.

16 X. L. Wang, X. P. Fu, W. W. Wang, C. Ma, R. Si and C. J. Jia, J. Phys. Chem. C, 2019, 123, 9001-9012.

17 X. Zhou, H. Y. Zhou, T. Y. Cheang, Z. W. Zhao, C. C. Shen, K. Liang, Y. N. Liu, Z. K. Yang, M. Imran and A. W. Xu, J. Phys. Chem. C, 2017, 121, 27528-27534.

18 K. Morgan, A. Goguet and C. Hardacre, ACS Catal., 2015, 5, 3430-3445.

19 Q. L. Zhu and Q. Xu, Chem, 2016, 1, 220-245.

20 Q. Yang, Q. Xu and H. L. Jiang, Chem. Soc. Rev., 2017, 46, 47744808.

21 V. Mouarrawis, R. Plessius, J. I. van der Vlugt and J. N. H. Reek, Front. Chem., 2018, 6.

22 S. Kitagawa, R. Kitaura and S. I. Noro, Angew. Chemie - Int. Ed., 2004, 43, 2334-2375.

23 S. Choi, M. O. Kee, O. M. Yaghi, S. L. James, O. M. Yaghi, H. Li, C. Davis, D. Richardson and T. L. Groy, 2003, 31, 474-484.

24 N. Stock and S. Biswas, Chem. Rev., 2012, 112, 933-969.

25 M. O'Keeffe and O. M. Yaghi, Chem. Rev., 2012, 112, 675-702.

26 H. Li, L. Li, R.-B. Lin, W. Zhou, Z. Zhang, S. Xiang and B. Chen, EnergyChem, 2019, 1, 100006. 
27 Y. He, W. Zhou, T. Yildirim and B. Chen, Energy Environ. Sci., 2013, 6, 2735-2744.

28 V. Pascanu, Q. Yao, A. Bermejo Gõmez, M. Gustafsson, Y. Yun, W. Wan, L. Samain, X. Zou and B. Martín-Matute, Chem. - A Eur. J., 2013, 19, 17483-17493.

29 S. H. Cho, B. Ma, S. B. T. Nguyen, J. T. Hupp and T. E. AlbrechtSchmitt, Chem. Commun., 2006, 2563-2565.

30 H. Wang, Q.-L. Zhu, R. Zou and Q. Xu, Chem, 2017, 2, 52-80.

31 K. M. Choi, H. M. Jeong, J. H. Park, Y. B. Zhang, J. K. Kang and O. M. Yaghi, ACS Nano, 2014, 8, 7451-7457.

32 M. A. Nasalevich, R. Becker, E. V. Ramos-Fernandez, S. Castellanos, S. L. Veber, M. V. Fedin, F. Kapteijn, J. N. H. Reek, J. I. Van Der Vlugt and J. Gascon, Energy Environ. Sci., 2015, 8, 364-375.

33 B. Li, H. M. Wen, H. Wang, H. Wu, M. Tyagi, T. Yildirim, W. Zhou and B. Chen, J. Am. Chem. Soc., 2014, 136, 6207-6210.

34 C. Rösler and R. A. Fischer, CrystEngComm, 2015, 17, 199-217.

35 J. Juan-Alcañiz, J. Gascon and F. Kapteijn, J. Mater. Chem., 2012, 22, 10102-10119.

36 M. Meilikhov, K. Yusenko, D. Esken, S. Turner, G. Van Tendeloo and R. A. Fischer, Eur. J. Inorg. Chem., 2010, 3701-3714.

37 K. Kočí, L. Obalová and Z. Lacný, Chem. Pap., 2008, 62, 1-9.

38 Y. B. Huang, M. Shen, X. Wang, P. Huang, R. Chen, Z. J. Lin and R. Cao, J. Catal., 2016, 333, 1-7.

39 M. Zhao, K. Yuan, Y. Wang, G. Li, J. Guo, L. Gu, W. Hu, H. Zhao and Z. Tang, Nature, 2016, 539, 76-80.

40 Y. Z. Chen, Z. U. Wang, H. Wang, J. Lu, S. H. Yu and H. L. Jiang, J. Am. Chem. Soc., 2017, 139, 2035-2044.

41 Y. Huang, Z. Zheng, T. Liu, J. Lü, Z. Lin, H. Li and R. Cao, Catal. Commun., 2011, 14, 27-31.

42 S. Campisi, M. Schiavoni, C. E. Chan-Thaw and A. Villa, Catalysts, 2016, 6.

43 D. E. Coupry, J. Butson, P. S. Petkov, M. Saunders, K. O'Donnell, H. Kim, C. Buckley, M. Addicoat, T. Heine and P. Á. Szilágyi, Chem. Commun., 2016, 52, 5175-5178.

44 B. Rungtaweevoranit, J. Baek, J. R. Araujo, B. S. Archanjo, K. M. Choi, O. M. Yaghi and G. A. Somorjai, Nano Lett., 2016, 16, 7645-7649.

45 N. Tsumori, L. Chen, Q. Wang, Q.-L. Zhu, M. Kitta and Q. Xu, Chem, 2018, 4, 845-856.

46 Y. Tong, G. Xue, H. Wang, M. Liu, J. Wang, C. Hao, X. Zhang, D. Wang, X. Shi, W. Liu, G. Li and Z. Tang, Nanoscale, 2018, 10, 16425-16430.

47 V. I. Isaeva, O. L. Eliseev, V. V. Chernyshev, T. N. Bondarenko, V. V. Vergun, G. I. Kapustin, A. L. Lapidus and L. M. Kustov, Polyhedron, 2019, 158, 55-64.

48 X. Li, T. W. Goh, L. Li, C. Xiao, Z. Guo, X. C. Zeng and W. Huang,
ACS Catal., 2016, 6, 3461-3468.

49 P. Szilágyi, D. M. Rogers, I. Zaiser, E. Callini, S. Turner, A. Borgschulte, A. Züttel, H. Geerlings, M. Hirscher and B. Dam, J. Mater. Chem. A, 2017, 5, 15559-15566.

50 A. Malouche, C. Zlotea and P. Á. Szilágyi, ChemPhysChem, 2019, 20, 1282-1295.

51 Q.-L. Zhu and Q. Xu, Chem, 2016, 1, 220-245.

52 A. Aijaz, A. Karkamkar, Y. J. Choi, N. Tsumori, E. Rönnebro, T. Autrey, H. Shioyama and Q. Xu, J. Am. Chem. Soc., 2012, 134, 13926-13929.

53 Y. K. Hwang, D. Y. Hong, J. S. Chang, S. H. Jhung, Y. K. Seo, J. Kim, A. Vimont, M. Daturi, C. Serre and G. Férey, Angew. Chemie Int. Ed., 2008, 47, 4144-4148.

54 Z.-W. Zhao, X. Zhou, Y.-N. Liu, C.-C. Shen, C.-Z. Yuan, Y.-F. Jiang, S.-J. Zhao, L.-B. Ma, T.-Y. Cheang and A.-W. Xu, Catal. Sci. Technol., 2018, 8, 3160-3165.

55 V. I. Isaeva, O. L. Eliseev, V. V. Chernyshev, T. N. Bondarenko, V. V. Vergun, G. I. Kapustin, A. L. Lapidus and L. M. Kustov, Polyhedron, 2019, 158, 55-64.

56 F. Fu, C. Wang, Q. Wang, A. M. Martinez-Villacorta, A. Escobar, H. Chong, X. Wang, S. Moya, L. Salmon, E. Fouquet, J. Ruiz and D. Astruc, J. Am. Chem. Soc., 2018, 140, 10034-10042.

57 S. Yoshimaru, M. Sadakiyo, A. Staykov, K. Kato and M. Yamauchi, Chem. Commun., 2017, 53, 6720-6723.

58 H. Zhao, H. Song and L. Chou, Inorg. Chem. Commun., 2012, 15, 261-265.

59 Z. Guo, C. Xiao, R. V. Maligal-Ganesh, L. Zhou, T. W. Goh, X. Li, D. Tesfagaber, A. Thiel and W. Huang, ACS Catal., 2014, 4, 13401348.

60 A. Aijaz, Q. L. Zhu, N. Tsumori, T. Akita and Q. Xu, Chem. Commun., 2015, 51, 2577-2580.

61 M. A. Addicoat, S. Fukuoka, A. J. Page and S. Irle, J. Comput. Chem., 2013, 34, 2591-2600.

62 M. J. Katz, Z. J. Brown, Y. J. Colón, P. W. Siu, K. A. Scheidt, R. Q. Snurr, J. T. Hupp and O. K. Farha, Chem. Commun., 2013, 49, 9449.

63 J. Teufel, H. Oh, M. Hirscher, M. Wahiduzzaman, L. Zhechkov, A. Kuc, T. Heine, D. Denysenko and D. Volkmer, Adv. Mater., 2013, 25, 635-639.

64 P. Á. Szilágyi, I. Weinrauch, H. Oh, M. Hirscher, J. Juan-Alcañiz, P. Serra-Crespo, M. De Respinis, B. J. Trześniewski, F. Kapteijn, H. Geerlings, J. Gascon, B. Dam, A. Grzech and R. Van De Krol, J. Phys. Chem. C, 2014, 118, 19572-19579.

65 H. scm. co. SCM, Theoretical Chemistry, Vrije Universiteit, Amsterdam, The Netherlands, Software for Chemistry and Materials, https://www.scm.com/.

66 J. M. Ducéré and L. Cavallo, J. Phys. Chem. B, 2007, 111, 1312413134. 
67 S. Grimme, J. Comput. Chem., 2004, 25, 1463-1473.

68 S. Grimme, J. Comput. Chem., 2006, 27, 1787-1799. 